\title{
Nonnutritive sweeteners and cardiometabolic health: a systematic review and meta-analysis of randomized controlled trials and prospective cohort studies
}

\author{
Meghan B. Azad PhD, Ahmed M. Abou-Setta MD PhD, Bhupendrasinh F. Chauhan MPharm PhD, \\ Rasheda Rabbani PhD, Justin Lys MD, Leslie Copstein MD, Amrinder Mann MD, Maya M. Jeyaraman MD PhD, \\ Ashleigh E. Reid MPAS, Michelle Fiander MLIS, Dylan S. MacKay PhD, Jon McGavock PhD, Brandy Wicklow MD MSc, \\ Ryan Zarychanski MD MSc
}

Cite as: CMAJ 2017 July 17;189:E929-39. doi: 10.1503/cmaj.161390

\begin{abstract}
BACKGROUND: Nonnutritive sweeteners, such as aspartame, sucralose and stevioside, are widely consumed, yet their long-term health impact is uncertain. We synthesized evidence from prospective studies to determine whether routine consumption of nonnutritive sweeteners was associated with long-term adverse cardiometabolic effects.
\end{abstract}

METHODS: We searched MEDLINE, Embase and Cochrane Library (inception to January 2016) for randomized controlled trials (RCTs) that evaluated interventions for nonnutritive sweeteners and prospective cohort studies that reported on consumption of nonnutritive sweeteners among adults and adolescents. The primary outcome was body mass index (BMI). Secondary outcomes included weight, obesity and other cardiometabolic end points.

RESULTS: From 11774 citations, we included 7 trials (1003 participants; median follow-up $6 \mathrm{mo}$ ) and 30 cohort studies (405 907 participants; median follow-up $10 \mathrm{yr}$ ). In the included RCTs, nonnutritive sweeteners had no significant effect on BMI (mean difference $-0.37 \mathrm{~kg} / \mathrm{m}^{2} ; 95 \%$ confidence interval [Cl] -1.10 to $0.36 ; I^{2} 9 \% ; 242$ participants). In the included cohort studies, consumption of nonnutritive sweeteners was associated with a modest increase in BMI (mean correlation 0.05, $95 \% \mathrm{Cl} 0.03$ to $0.06 ; I^{2} 0 \% ; 21256$ participants). Data from RCTs showed no consistent effects of nonnutritive sweeteners on other measures of body composition and reported no further secondary outcomes. In the cohort studies, consumption of nonnutritive sweeteners was associated with increases in weight and waist circumference, and higher incidence of obesity, hypertension, metabolic syndrome, type 2 diabetes and cardiovascular events. Publication bias was indicated for studies with diabetes as an outcome.

INTERPRETATION: Evidence from RCTS does not clearly support the intended benefits of nonnutritive sweeteners for weight management, and observational data suggest that routine intake of nonnutritive sweeteners may be associated with increased BMI and cardiometabolic risk. Further research is needed to fully characterize the long-term risks and benefits of nonnutritive sweeteners. Protocol registration: PROSPERO-CRD42015019749

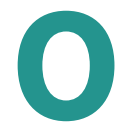

besity is a major public health challenge that contributes to type 2 diabetes and cardiovascular disease. ${ }^{1}$ Evidence that sugar consumption is fuelling this epidemic $^{2-4}$ has stimulated the increasing popularity of nonnutritive sweeteners, ${ }^{5}$ including aspartame, sucralose and stevioside. In 2008 , more than $30 \%$ of Americans reported daily intake of nonnutritive sweeteners, and this proportion is increasing. ${ }^{6}$ Researchers have suggested that nonnutritive sweeteners may have adverse effects on glucose metabolism, gut microbiota and appetite control. ${ }^{7,8}$ Moreover, studies involving animals have reported that chronic exposure to nonnutritive sweeteners leads to increased food consumption, weight gain and adiposity. ${ }^{9}$

The position of the Academy of Nutrition and Dietetics is that nonnutritive sweeteners can help limit energy intake as a strategy to manage weight or blood glucose. ${ }^{10}$ However, consumption of nonnutritive sweeteners has been paradoxically associated 
with weight gain and incident obesity. ${ }^{7,11} \mathrm{~A}$ previous metaanalysis ${ }^{12}$ reported conflicting evidence: randomized controlled trials (RCTs) showed potential benefits (modest weight loss), whereas observational studies showed a small but significant association with increased body mass index (BMI). However, the review did not evaluate outcomes beyond body composition. ${ }^{13}$ Several studies involving more than 100000 new participants and representing several new geographic settings have since been published. ${ }^{14-24}$

Our objective was to synthesize evidence addressing this question: Is routine consumption of nonnutritive sweeteners by adults and adolescents associated with adverse long-term cardiometabolic effects in RCTs and prospective cohort studies?

\section{Methods}

This review is reported according to the Preferred Reporting Items for Systematic Reviews and Meta-Analyses ${ }^{25}$ following a registered protocol. ${ }^{26}$

\section{Search strategy and selection criteria}

The search strategy was developed by an information specialist (M.F.) to overcome the limitations ${ }^{13}$ of previous reviews. Our MEDLINE strategy (Appendix 1, available at www.cmaj.ca/lookup /suppl/doi:10.1503/cmaj.161390/-/DC1, Table S1) was peer reviewed and also translated for searches in Embase and The Cochrane Central Register of Controlled Trials. We included the following terms, among others: nonnutritive sweeteners, aspartame, saccharin, sucralose, xylitol, stevia, carbonated beverages, calories and food frequency. We did not limit the search by using terms related to outcomes of interest.

We conducted the searches from the time of database inception to January 2016 with no language restrictions; translation services were accessed to evaluate non-English citations. We also searched conference proceedings from the American Society for Nutrition, American Diabetes Association and Obesity Society. We manually searched reference lists of pertinent reviews and included studies for relevant citations, and we conducted grey literature searches of OpenSIGLE and Google Scholar. We used EndNote (version X6, Thompson Reuters, New York) to perform reference management.

We screened search results in duplicate using a team of 5 reviewers (A.M., A.R., J.L., L.C., M.J.). We included RCTs and observational studies that evaluated consumption of nonnutritive sweeteners in individuals who were more than 12 years of age (Appendix 1, Table S2). Studies evaluating children were reviewed separately. ${ }^{27}$ We required a minimum study duration of 6 months to reflect routine consumption of nonnutritive sweeteners, to focus on long-term effects and to allow time for metabolic outcomes to develop. For observational studies, we required that associations with baseline intake of nonnutritive sweeteners (not only changes in intake during the course of the study) were reported to confirm temporality and limit confounding by reverse causation. Our primary outcome was change in BMI.

Secondary outcomes included changes in body weight; adiposity; glucose metabolism; and incidence of overweight/obesity, metabolic syndrome, type 2 diabetes, hypertension and other cardiorenal outcomes. If a study reported outcomes at multiple time points, we included the longest available follow-up.

\section{Data extraction}

We developed, piloted and deployed a standardized form for data extraction in DistillerSR (version 2, Evidence Partners Inc., Ottawa). A team of 5 reviewers (A.A., B.C., R.R., L.C., M.A.) independently extracted study data in duplicate that included baseline characteristics; interventions for nonnutritive sweeteners and comparators (for trials) or consumption of nonnutritive sweeteners and confounders or covariates (for cohorts); type, dose and duration of exposure to nonnutritive sweeteners; duration of follow-up; and cardiometabolic outcomes. For RCTs, we preferentially extracted data from intention-to-treat analyses or requested the data from authors. For cohorts, we extracted adjusted effect estimates in 2 formats: ratios comparing the highest versus lowest category of nonnutritive sweetener intake, and beta estimates quantifying linear associations per unit of nonnutritive sweetener intake. If multiple adjusted estimates were reported, we extracted the estimate from the statistical model that included the largest number of covariates. Data that were presented in nonextractable formats were requested from authors.

\section{Assessment of study quality}

Four reviewers (M.A., J.L., L.C., B.C.) assessed potential bias in RCTs using the Cochrane Collaboration Risk of Bias tool ${ }^{29,30}$ and evaluated the quality of cohort studies using the 9-point NewcastleOttawa Scale. ${ }^{31}$ Based on previous research ${ }^{32,33}$ we designated 2 critical confounders for cohort studies: baseline body composition (BMI or other measure of body composition) and diet quality (total energy or sugar intake, or a diet pattern or quality score).

\section{Statistical analysis}

For the meta-analysis of continuous outcomes, we calculated mean differences (MD) or standardized MDs. For binary outcomes, we calculated pooled odds ratios (ORs), risk ratios (RRs) or hazard ratios (HRs), and 95\% confidence intervals (Cls). When nonnutritive sweetener intake units differed between cohort studies, we converted $\beta$ estimates to $t$ values ( $\beta /$ standard error) to generate a unitless metric ${ }^{28}$ and calculated the pooled mean correlation. Subgroup analyses were planned a priori to explore heterogeneity and determine associations in prespecified strata. We conducted the analyses with random-effects models using Comprehensive Meta-Analysis Software (version 2.2.064) or RevMan (version 5.3.5). Statistical heterogeneity was quantified using the $I^{2}$ statistic. We assessed publication bias using funnel plots, and the trim and fill method.

\section{Results}

From 11774 citations, we assessed 938 full-text articles for eligibility, and 37 studies involving a total of 406910 individuals met our inclusion criteria: 7 RCTs R $^{19,20,34-38}$ and 30 cohort studies $^{14-18,21-24,39-60}$ (Figure 1). 
The 7 RCTs enrolled a total of 1003 participants who were obese, ${ }^{38}$ overweight $^{19,20,34,35}$ or hypertensive ${ }^{36,37}$ (Table 1 ). The interventions for nonnutritive sweeteners included beverages sweetened with aspartame or unspecified nonnutritive sweeteners, ${ }^{19,20,34,35}$ stevioside capsules ${ }^{36,37}$ or consumption of aspartame at the discretion of the participant. ${ }^{38}$ The duration of interventions ranged from 6 to 24 months (median 6 mo, interquartile range [IQR] 6-14). Most RCTs were at unclear or high risk of bias (Table 1 and Appendix 1, Table S3).

The 30 observational studies reported outcomes from 22 distinct cohorts involving a total of 405907 individuals (Table 2). Most of the studies used food frequency questionnaires to evaluate beverages containing nonnutritive sweeteners. More than $85 \%$ controlled for baseline body composition, diet quality, age, sex, smoking and physical activity, whereas less than 50\% controlled for ethnicity and socioeconomic status (Appendix 1, Table S4). The duration of follow-up ranged from 1 to 38 years (median $10 \mathrm{yr}$, IQR 6-22). Most cohort studies were of moderate quality (Table 2 and Appendix 1, Table S5).

\section{Primary outcome: body mass index}

Two RCTs involving hypertensive participants who were taking stevioside capsules $^{36,37}$ and 1 RCT involving participants who were overweight and consuming artificially sweetened beverages $^{20}$ showed no significant effect on BMI over 6 to 24 months (MD $-0.37 \mathrm{~kg} /$ $\mathrm{m}^{2}, 95 \% \mathrm{Cl}-1.10$ to 0.36 ; $1^{2}$ 9\%; 3 trials; 242 participants; Table 3, Figure 2A). Two cohort studies that reported continuous nonnutritive sweetener intake in healthy participants ${ }^{14,15}$ showed a positive correlation with BMI over 3 to 13 years (mean correlation $0.05,95 \% \mathrm{Cl}$ 0.03 to $0.06 ; I^{2} 0 \%$; 2 cohorts; 21256 participants; Table 3, Figure 2B). A third cohort study that reported quantiles of nonnutritive sweetener intake ${ }^{50}$ found that participants who consumed nonnutrive sweeteners daily had a greater increase in BMI during 8 years of follow-up than those who did not consume them (MD $0.77 \mathrm{~kg} / \mathrm{m}^{2}, 95 \% \mathrm{Cl}$ 0.47 to 1.07 for daily v. no intake; 3371 participants). Overall, there was limited evidence for the effect of nonnutritive sweeteners on BMI, with 3 longterm cohort studies suggesting a modest increase in BMI that was not confirmed in 2 RCTs. The limited number of eligible studies precluded subgroup analyses.

\section{Secondary outcomes}

\section{Weight}

Among 5 RCTs evaluating interventions using nonnutritive sweeteners in participants who were obese, ${ }^{19,20,34,35,38}$ there was no consistent effect on change in weight (standardized MD -0.17; $95 \% \mathrm{Cl}-0.54$ to $0.21 ; P^{2} 81 \%$; 5 trials; 791 participants) (Table 3, Figure $2 \mathrm{C}$ ). Heterogeneity across the 5 trials was partially explained by differences in study duration: 2 longer trials ${ }^{19,38}$ showed significant weight loss over 16 to 24 months of the intervention (standardized MD $-0.55,95 \% \mathrm{Cl}-0.75$ to $-0.34 ; 1^{2} 0 \% ; 2$ trials), and 3 shorter ( $6 \mathrm{mo}$ ) trials $\mathrm{s}^{20,34,35}$ showed no effect for the use of nonnutritive sweeteners (standardized MD $0.13,95 \% \mathrm{Cl}$ -0.34 to $0.59 ; I^{2} 65 \% ; 3$ trials) ( $p$ for subgroup differences $=0.009$; Appendix 1, Table S6). Weight-loss effects also tended to be
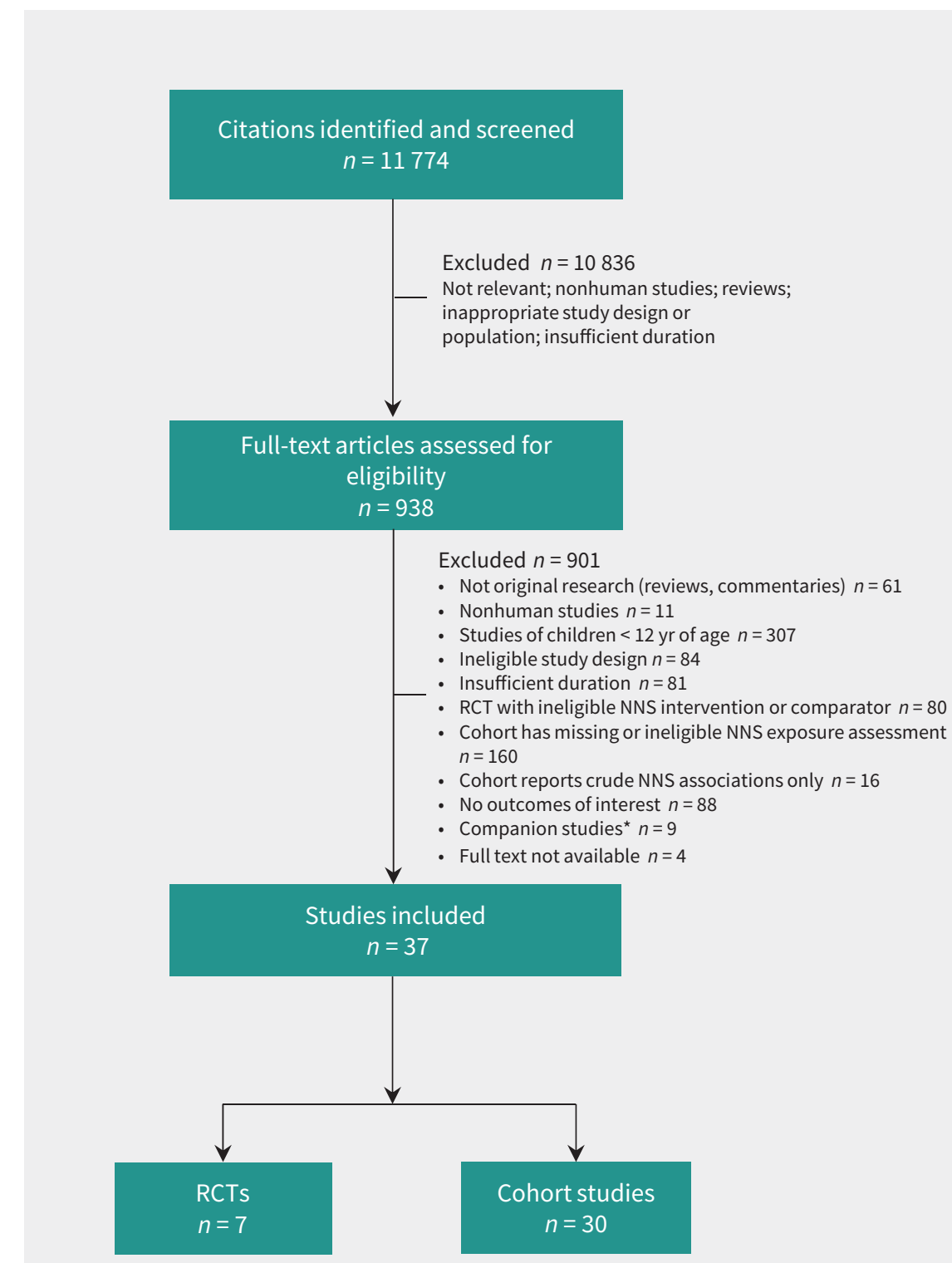

Figure 1: PRISMA flow diagram. NNS = nonnutritive sweetener, RCT = randomized controlled trial. ${ }^{\star}$ Companion studies included abstracts, trial registrations and earlier reports from included studies. 
Table 1: Randomized controlled trials that evaluated nonnutritive sweetener interventions and long-term cardiometabolic health

\begin{tabular}{|c|c|c|c|c|c|c|c|c|c|c|c|c|c|}
\hline $\begin{array}{l}\text { Study, } \\
\text { country }\end{array}$ & $\begin{array}{l}\text { No. of } \\
\text { participants } \\
\text { randomly } \\
\text { assigned (\% } \\
\text { completed) }\end{array}$ & Sex & Population & $\begin{array}{c}\text { Age, } \\
\text { mean } \pm \text { SD } \\
y r\end{array}$ & $\begin{array}{c}\text { BMI, } \\
\text { mean } \pm \text { SD; } \\
\text { kg/m² }\end{array}$ & $\begin{array}{c}\text { Duration, } \\
\text { mo }\end{array}$ & $\begin{array}{c}\text { Type and } \\
\text { source of } \\
\text { NNS }\end{array}$ & $\begin{array}{c}\text { Daily dose of } \\
\text { NNS }\end{array}$ & $\begin{array}{l}\frac{\pi}{4} \\
\frac{0}{0} \\
\frac{0}{\pi} \\
\frac{0}{E} \\
\frac{0}{0}\end{array}$ & $\sum_{\infty}$ & 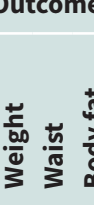 & 范 $\frac{\alpha}{\overline{1}}$ & $\begin{array}{l}\frac{1}{n} \\
\frac{\pi}{0} \\
\frac{0}{0} \\
\frac{x}{u} \\
\frac{u}{x}\end{array}$ \\
\hline $\begin{array}{l}\text { Blackburn et } \\
\text { al. } 1997,{ }^{38} \\
\text { USA }\end{array}$ & $163(53)$ & $\mathrm{F}$ & $\begin{array}{l}\text { Obese, on } \\
\text { weight-loss } \\
\text { program }\end{array}$ & $44 \pm 10$ & $37 \pm 5$ & 16 & $\begin{array}{l}\text { Aspartame } \\
\text { ASB, packets, } \\
\text { foodstuffs }\end{array}$ & $\begin{array}{l}\text { Participants' } \\
\text { discretion }\end{array}$ & $\begin{array}{l}\text { Aspartame } \\
\text { avoidance }\end{array}$ & & - & & High \\
\hline $\begin{array}{l}\text { Hsieh et al. } \\
2003,{ }^{36} \text { China }\end{array}$ & $174(97)$ & $\mathrm{M}, \mathrm{F}$ & $\begin{array}{c}\text { Mild } \\
\text { hypertension }\end{array}$ & $52 \pm 7$ & $23 \pm 3$ & 24 & $\begin{array}{l}\text { Stevioside } \\
\text { capsules }\end{array}$ & $1500 \mathrm{mg}$ & Placebo & $\bullet$ & & & Low \\
\hline $\begin{array}{l}\text { Ferri et al. } \\
2006,{ }^{37} \text { Brazil }\end{array}$ & $14(86)$ & $M, F$ & $\begin{array}{c}\text { Mild } \\
\text { hypertension }\end{array}$ & $45 \pm 7$ & $27 \pm 3$ & 6 & $\begin{array}{l}\text { Stevioside } \\
\text { capsules }\end{array}$ & $\begin{array}{l}3 \text { phases: } 3.8 \text {, } \\
7.5,15.0 \mathrm{mg} / \mathrm{kg}\end{array}$ & Placebo & $\bullet$ & & • & Unclear \\
\hline $\begin{array}{l}\text { Tate et al. } \\
2012,{ }^{34} \text { USA }\end{array}$ & $213(86)$ & $\mathrm{M}, \mathrm{F}$ & $\begin{array}{c}\text { Overweight, on } \\
\text { weight-loss } \\
\text { program }\end{array}$ & $42 \pm 11$ & $36 \pm 6$ & 6 & $\begin{array}{c}\text { Unspecified } \\
\text { ASB }\end{array}$ & $\begin{array}{c}\text { Recommended } \\
\geq 2 \text { servings }\end{array}$ & $\begin{array}{l}\text { Water, } \\
\text { attention } \\
\text { control }\end{array}$ & & • • & & High \\
\hline $\begin{array}{l}\text { Maersk et al. } \\
2012,{ }^{35} \\
\text { Denmark }\end{array}$ & $33(76)$ & $\mathrm{M}, \mathrm{F}$ & Overweight & $39 \pm 8$ & $33 \pm 4$ & 6 & $\begin{array}{c}\text { Aspartame } \\
\text { ASB }\end{array}$ & $1 \mathrm{~L}$ of diet cola & Water & & - & • & High \\
\hline $\begin{array}{l}\text { Peters et al. } \\
2016,{ }^{19} \text { USA }\end{array}$ & $308(72)$ & $\mathrm{M}, \mathrm{F}$ & $\begin{array}{c}\text { Overweight, on } \\
\text { weight-loss } \\
\text { program }\end{array}$ & $48 \pm 11$ & $34 \pm 4$ & 12 & $\begin{array}{c}\text { Unspecified } \\
\text { ASB }\end{array}$ & $\begin{array}{l}\text { At least } \\
710 \mathrm{~mL}\end{array}$ & $\begin{array}{l}\text { Water with ASB } \\
\text { avoidance }\end{array}$ & & • • & & High \\
\hline $\begin{array}{l}\text { Madjd et al. } \\
2015,{ }^{20} \text { Iran }\end{array}$ & $71(87)$ & $\mathrm{F}$ & $\begin{array}{c}\text { Overweight, on } \\
\text { weight-loss } \\
\text { program }\end{array}$ & $32 \pm 7$ & $34 \pm 3$ & 6 & $\begin{array}{c}\text { Unspecified } \\
\text { ASB }\end{array}$ & $250 \mathrm{~mL}$ & Water & & • • & $\bullet$ & High \\
\hline
\end{tabular}

stronger in RCTs with industry sponsorship ${ }^{19,34,38}$ (standardized MD $-0.37 ; 95 \% \mathrm{Cl}-0.71$ to $-0.03 ; l^{2} 77 \%$; 3 trials) compared with RCTs that were not funded by industry ${ }^{20,35}$ (standardized MD 0.30 , $95 \% \mathrm{Cl}-0.38$ to $0.99 ; I^{2} 55 \% ; 2$ trials) ( $p$ for subgroup differences $=$ 0.09; Appendix 1, Table S6). Notably, both longer-term RCTs were funded by industry, ${ }^{19,38}$ making it impossible to isolate the effect of trial duration and industry sponsorship in subgroup analyses. In addition, all 5 RCTs that evaluated weight change were at high risk of bias, prohibiting subgroup analyses according to this metric.

Two observational studies reported on intake of nonnutritive sweeteners and subsequent weight change in 4 cohorts over periods of 2 to 4 years ${ }^{21,57}$ (Table 3, Figure 2D). There was a significant positive correlation between intake of nonnutritive sweeteners and weight gain (weighted mean correlation $0.06,95 \% \mathrm{Cl}$ 0.05 to 0.07 ; $I^{2} 46 \%$; 4 cohorts; 32405 participants) (Table 3).

\section{Adiposity and overweight}

Three RCTs involving participants who were obese and consuming diet soda as part of a weight-loss program reported inconsistent effects on waist circumference (standardized MD -0.16; 95\% Cl -0.56 to $0.25 ; P^{2}$ 83\%; 3 trials; 683 participants) (Table 3, Appendix 1, Figure S1A). Heterogeneity across studies was related to the duration of intervention, with one 12-month trial showing a significant reduction in waist circumference ${ }^{19}$ and two 6 -month interventions finding no effect ${ }^{20,34}$ ( $p$ for subgroup differences 0.001 ). One 6-month trial reported no effect on percentage of body fat. ${ }^{35}$

In contrast to RCTs, cohort studies with 4 to 9 years of followup showed that higher intake of nonnutritive sweeteners was associated with increasing waist circumference (MD $2.27 \mathrm{~cm}$, 95\% Cl 0.96 to 3.58; 1 cohort; 384 participants) ${ }^{18}$ (Table 3), higher incidence of abdominal obesity (OR $1.59,95 \% \mathrm{Cl} 1.23$ to 2.07; 1 cohort; 5011 participants) ${ }^{60}$ (Table 3) and higher incidence of overweight (OR $1.84,95 \% \mathrm{Cl} 1.28$ to 2.66 for highest v. lowest intake quantiles; $I^{2} 0 \%$; 3 cohorts; 7917 participants) ${ }^{22,50,59}$ (Table 3 and Appendix 1, Figure S1B).

\section{Metabolic outcomes}

Incidence for metabolic syndrome and type 2 diabetes was not reported in the RCTs. Pooled data from cohort studies with 4 to 24 years of follow-up showed higher risk of metabolic syndrome (RR $1.31,95 \% \mathrm{Cl} 1.23$ to $1.40 ; I^{2}$ 0\%; 5 cohorts; 27914 participants) $39,47,48,54,60$ (Table 3 and Appendix 1, Figure S2A) and type 2 diabetes (RR 1.14, 95\% Cl 1.05 to 1.25; 2 52\%; 9 cohorts; 400571 participants) ${ }^{16,24,42,49,55,56,58,60}$ for the highest versus lowest quan- 


\begin{tabular}{|c|c|c|c|c|c|c|c|c|c|c|c|c|c|c|c|c|}
\hline Study* & Cohort & $\begin{array}{c}\text { Country, } \\
\text { year of } \\
\text { baseline } \\
\text { NNS } \\
\text { intake }\end{array}$ & $\begin{array}{c}\text { No. of } \\
\text { participants }\end{array}$ & Sex & $\begin{array}{c}\text { Age at } \\
\text { baseline, } \\
\text { mean } \pm \\
\text { SD, or } \\
\text { range; yr }\end{array}$ & $\begin{array}{c}\text { BMI at } \\
\text { baseline, } \\
\text { mean } \pm \text { SD, } \\
\text { or } \% \text { OW; } \\
\mathrm{kg} / \mathrm{m}^{2}\end{array}$ & $\begin{array}{c}\text { Follow- } \\
\text { up, yr }\end{array}$ & $\begin{array}{l}\text { Type or } \\
\text { source of } \\
\text { NNS }\end{array}$ & $\begin{array}{l}\text { Extreme NNS } \\
\text { intake } \\
\text { categories, } \\
\text { servings } \dagger\end{array}$ & $\begin{array}{l}\text { Measure of } \\
\text { continuous } \\
\text { NNS intake }\end{array}$ & $\sum_{\infty}$ & 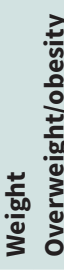 & 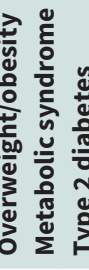 & 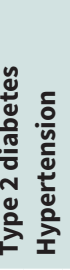 & $\frac{1}{0}$ & 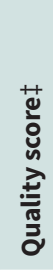 \\
\hline $\begin{array}{l}\text { Lutsey et al. } \\
2008^{54}\end{array}$ & ARIC & USA, 1987 & 9154 & $M, F$ & $54 \pm 6$ & - & 9 & AS soda & Extreme tertiles & - & & & - & & & 8 \\
\hline $\begin{array}{l}\text { Bomback et al. } \\
2010^{43}\end{array}$ & ARIC & USA, 1987 & 14002 & $\mathrm{M}, \mathrm{F}$ & $54 \pm 6$ & $28 \pm 5$ & 9 & AS soda & $>1 / \mathrm{d} v .<1 / \mathrm{d}$ & - & & & & & CKD & 9 \\
\hline $\begin{array}{l}\text { Palmer et al. } \\
2008^{55}\end{array}$ & BWHS & USA, 2001 & 43960 & $\mathrm{~F}$ & $38 \pm 10$ & $28 \pm 7$ & 4 & AS soda & $\geq 1 / \mathrm{d} \mathrm{v} .<1 / \mathrm{mo}$ & - & & & • & • & & 6 \\
\hline $\begin{array}{l}\text { Duffey et al. } \\
2012^{48}\end{array}$ & CARDIA & USA, 1986 & 3728 & $M, F$ & $25 \pm 26$ & $25 \pm 5$ & 20 & ASB & None $v$. any & - & & & • & • & IGT & 8 \\
\hline $\begin{array}{l}\text { Haines et al. } \\
2007^{59}\end{array}$ & EAT & USA, 1998 & 2516 & $M, F$ & $15 \pm 2$ & $11 \%$ OW & 5 & AS soda & - & serving/d & & & • & & & 7 \\
\hline $\begin{array}{l}\text { Lana et al. } \\
2015 \S^{22}\end{array}$ & ENRICA & Spain, 2008 & 2030 & $\mathrm{M}, \mathrm{F}$ & $18-60$ & $26 \pm 5$ & 4 & AS soda & $\geq 1 /$ d v. $<1 / w k$ & - & & • & - & & & 9 \\
\hline $\begin{array}{l}\text { Fagherazzi et } \\
\text { al. 2013 } \text { | }^{49}\end{array}$ & EPIC-E3N F & France, 1993 & 66118 & $\mathrm{~F}$ & $53 \pm 7$ & $19 \%$ OW & 17 & ASB & $\begin{array}{c}>603 \mathrm{~mL} / \mathrm{wk} \mathrm{v} . \\
\text { never }\end{array}$ & - & & & & • & & 8 \\
\hline $\begin{array}{l}\text { O'Connor et al. } \\
\left.2015\right|^{24}\end{array}$ & $\begin{array}{l}\text { EPIC- } \\
\text { Norfolk }\end{array}$ & UK, 1993 & 24653 & $M, F$ & $58 \pm 9$ & $26 \pm 4$ & 11 & ASB & $\begin{array}{l}\geq 169 \mathrm{~mL} / \mathrm{d} \mathrm{v} . \\
\text { none }\end{array}$ & serving/d & & & & • & & 8 \\
\hline $\begin{array}{l}\text { Dhingra et al. } \\
2007^{47}\end{array}$ & FOS & USA, 1992 & 1864 & $M, F$ & $55 \pm 10$ & $27 \pm 5$ & 4 & AS soda & $1 / \mathrm{d} v .<1 / w k$ & - & & & - & & & 9 \\
\hline $\begin{array}{l}\text { Field et al. } \\
2014^{14}\end{array}$ & GUTS II & USA, 2004 & 7559 & $M, F$ & $13 \pm 2$ & $20 \pm 3$ & 3 & AS soda & - & serving/d & $\bullet$ & & & & & 6 \\
\hline $\begin{array}{l}\text { Bernstein et al. } \\
2012^{40}\end{array}$ & HPFS & USA, 1986 & 43371 & M & $62 \pm 11$ & $26 \pm 3$ & 22 & AS soda & $\geq 1 / d v$. none & serving /d & & & & & Stroke & 8 \\
\hline $\begin{array}{l}\text { Bhupathiraju et } \\
\text { al. } 2013^{\star \star 42}\end{array}$ & $t$ HPFS & USA, 1986 & 39059 & M & $53 \pm 10$ & $25 \pm 5$ & 22 & AS soda & $\geq 1 / \mathrm{d} \mathrm{v} .<1 / \mathrm{mo}$ & serving/d & & & & • & & 7 \\
\hline $\begin{array}{l}\text { Cohen et al. } \\
2012^{45}\end{array}$ & HPFS & USA, 1986 & 37360 & M & $40-75$ & $25 \pm 3$ & 22 & ASB & $\geq 1 / \mathrm{d} \mathrm{v} .<1 / \mathrm{mo}$ & - & & & & - & & 8 \\
\hline $\begin{array}{l}\text { de Koning et al. } \\
2012^{46}\end{array}$ & . HPFS & USA, 1986 & 42883 & M & $40-75$ & $26 \pm 3$ & 22 & ASB & > 4/wk v. none & serving/d & & & & & CHD & 8 \\
\hline $\begin{array}{l}\text { Smith et al. } \\
2015^{21}\end{array}$ & HPFS & USA, 1986 & 21472 & M & $47 \pm 6$ & $25 \pm 1$ & 24 & AS soda & - & serving/d & & - & & & & 6 \\
\hline $\begin{array}{l}\text { Gearon et al. } \\
2014 \S^{15}\end{array}$ & MCCS & $\begin{array}{c}\text { Australia, } \\
1990\end{array}$ & 13697 & $\mathrm{M}, \mathrm{F}$ & $55 \pm 9$ & $26 \pm 4$ & 13 & AS soda & - & serving/wk & • & & & & & 8 \\
\hline $\begin{array}{l}\text { Nettleton et al. } \\
2009^{60}\end{array}$ & MESA & USA, 2000 & 5011 & $\mathrm{M}, \mathrm{F}$ & $62 \pm 11$ & $28 \pm 6$ & 5 & AS soda & $\begin{array}{c}\geq 1 / \mathrm{d} \text { v. rare or } \\
\text { never }\end{array}$ & - & & & - • & • $\bullet$ & Waist & 6 \\
\hline $\begin{array}{l}\text { Fung et al. } \\
2009^{51}\end{array}$ & NHS I & USA, 1980 & 88520 & $\mathrm{~F}$ & $34-59$ & $24 \pm 2$ & 24 & AS soda & $\geq 2 / \mathrm{d} \mathrm{v} .<1 / \mathrm{mo}$ & - & & & & & CHD & 8 \\
\hline $\begin{array}{l}\text { Bernstein et al. } \\
2012^{40}\end{array}$ & NHS I & USA, 1980 & 84085 & $\mathrm{~F}$ & $58 \pm 10$ & $26 \pm 5$ & 28 & AS soda & $\geq 1 / d v$. none & serving /d & & & & & Stroke & 8 \\
\hline $\begin{array}{l}\text { Bhupathiraju et } \\
\text { al. } 2013^{42}\end{array}$ & N NHSI & USA, 1984 & 74749 & $\mathrm{~F}$ & $50 \pm 7$ & $25 \pm 5$ & 24 & AS soda & $\geq 1 / \mathrm{d} \mathrm{v} .<1 / \mathrm{mo}$ & serving/d & & & & • & & 7 \\
\hline $\begin{array}{l}\text { Cohen et al. } \\
2012 t^{45}\end{array}$ & NHSI & USA, 1980 & 88540 & $\mathrm{~F}$ & $34-59$ & $23 \pm 3$ & 38 & ASB & $\geq 1 / \mathrm{d} \mathrm{v} .<1 / \mathrm{mo}$ & - & & & & $\bullet$ & & 8 \\
\hline $\begin{array}{l}\text { Smith et al. } \\
2015 \ddagger \ddagger^{21}\end{array}$ & NHS I & USA, 1986 & 48449 & $\mathrm{~F}$ & $49 \pm 6$ & $24 \pm 1$ & 24 & AS soda & - & serving/d & & - & & & & 6 \\
\hline $\begin{array}{l}\text { Pan et al. } \\
2012 \S \S^{56}\end{array}$ & NHS II & USA, 1991 & 82902 & $\mathrm{~F}$ & $36 \pm 5$ & $24 \pm 5$ & 18 & ASB & $\geq 4 / \mathrm{d} v . \leq 1 / w k$ & serving/d & & & & • & & 7 \\
\hline $\begin{array}{l}\text { Chen et al. } \\
2009^{44}\end{array}$ & NHS II & USA, 1991 & 13475 & $\mathrm{~F}$ & $32 \pm 3$ & $23 \pm 4$ & 10 & ASB & $\geq 5 / \mathrm{wk} \mathrm{v} . \leq 3 / \mathrm{mo}$ & o serving/d & & & & & GDM & 8 \\
\hline $\begin{array}{l}\text { Cohen et al. } \\
2012 \dagger^{45}\end{array}$ & NHS II & USA, 1991 & 97991 & $\mathrm{~F}$ & $27-42$ & $23 \pm 4$ & 16 & ASB & $\geq 1 / \mathrm{d} \mathrm{v} .<1 / \mathrm{mo}$ & - & & & & • & & 8 \\
\hline $\begin{array}{l}\text { Smith et al. } \\
2015^{21}\end{array}$ & NHS II & USA, 1991 & 48071 & $\mathrm{~F}$ & $38 \pm 4$ & $23 \pm 2$ & 16 & AS soda & - & serving/d & & - & & & & 6 \\
\hline
\end{tabular}




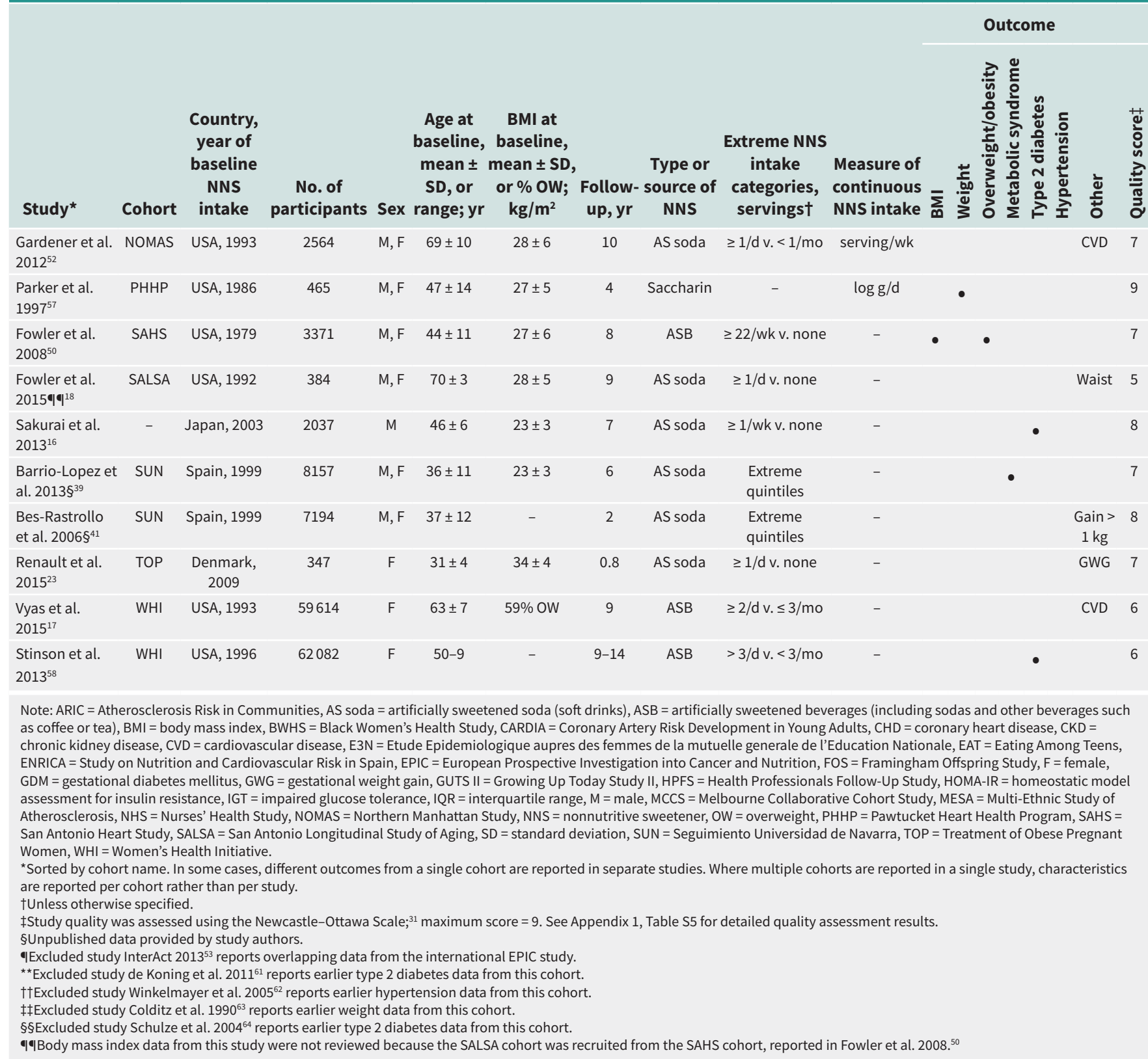

tiles of nonnutritive sweetener intake (Table 3, Figure 2E). In subgroup analyses, heterogeneity was not explained by baseline weight status, study quality, duration of follow-up or dose of nonnutritive sweeteners (Appendix 1, Table S7). Among 4 cohorts that reported continuous effect estimates, we found a $3 \%$ higher relative risk of type 2 diabetes per additional daily serving of nonnutritive sweetener (RR $1.03,95 \% \mathrm{Cl} 1.01$ to $1.05 ; I^{2} 0 \%$; 4 cohorts; 221363 participants) ${ }^{24,42,53,56}$ (Table 3 and Appendix 1, Figure S2B). We found no statistically significant associations for insulin resistance (3 trials; Appendix 1, Figure S3), glycosylated hemoglobin (1 trial), glucose tolerance (1 cohort) or gestational diabetes (1 cohort) (Table 3).

\section{Cardiorenal outcomes}

Cardiorenal outcomes were not reported in the RCTs. Among cohort studies, we found that high nonnutritive sweetener intake was associated with a higher risk of hypertension over 5 to 38 years of follow-up (HR $1.13,95 \%$ Cl 1.06 to $1.20 ; I^{2} 64 \%$; 5 cohorts; 232630 participants) ${ }^{45,48,60}$ (Table 3 and Appendix 1, Figure $\mathrm{S} 4 \mathrm{~A})$. In addition, high intake of nonnutritive sweetener was associated with a higher risk of stroke (RR $1.14,95 \% \mathrm{Cl} 1.04$ to 1.26; $l^{2}$ 0\%; 2 cohorts; 128176 participants $)^{40}$ and cardiovascular events (RR 1.32; $95 \% \mathrm{Cl} 1.15$ to $1.52 ; \mathrm{I}^{2}$ 0\%; 2 cohorts; 62178 participants), ${ }^{17,52}$ whereas there was no significant association with coronary heart disease (RR $0.98 ; 95 \% \mathrm{Cl} 0.90$ to $1.07 ; I^{2} 0 \%$; 
Table 3: Results from meta-analyses (where possible) or individual studies for intake of nonnutritive sweeteners and

long-term cardiometabolic health outcomes in randomized controlled trials and cohort studies

\begin{tabular}{|c|c|c|c|c|c|c|}
\hline $\begin{array}{l}\text { Outcome: } \\
\text { change or incidence }\end{array}$ & $\begin{array}{c}\text { No. of } \\
\text { studies* } \\
\text { (participants) }\end{array}$ & Comparison & $\begin{array}{l}\text { Estimate of NNS effect }(95 \% \mathrm{Cl}) \\
\text { from meta-analysis or individual } \\
\text { studies }\end{array}$ & Assoc. & Citation(s)* & Figure \\
\hline \multicolumn{7}{|l|}{ Randomized controlled trials } \\
\hline BMI & $3(242)$ & NNS v. control & $\mathrm{MD}-0.37 \mathrm{~kg} / \mathrm{m}^{2}$ (-1.10 to 0.36$), \perp^{2} 9 \%$ & NS & $20,36,37$ & 2 \\
\hline Weight & $5(791)$ & NNS v. control & SMD $-0.17(-0.54$ to 0.21$), I^{2} 81 \%$ & NS & $19,20,34,35,38$ & 2 \\
\hline Percentage of fat mass & $1(25)$ & NNS v. control & MD $-1.01 \%(-3.01$ to 0.99$)$ & NS & 35 & - \\
\hline Waist circumference & $3(683)$ & NNS v. control & SMD $-0.16(-0.56$ to 0.25$), 1^{2} 83 \%$ & NS & $19,20,34$ & $\mathrm{~S} 1 \ddagger$ \\
\hline Insulin resistance: HOMA-IR & $3(99)$ & NNS v. control & SMD +0.10 (-0.57 to 0.76$), I^{2} 55 \%$ & NS & $20,35,37$ & S3 \\
\hline $\mathrm{HbA}_{1 \mathrm{c}}$ & $1(62)$ & NNS v. control & $\mathrm{MD}+0.07 \%(-0.00$ to 0.14$)$ & NS & 20 & - \\
\hline \multicolumn{7}{|l|}{ Cohort studies } \\
\hline \multirow[t]{2}{*}{ BMI } & $2(21256)$ & Continuous correlation & WMC $+0.05(0.03$ to 0.06$), I^{2} 0 \%$ & $\uparrow$ Gain & 14,15 & 2 \\
\hline & $1(3371)$ & $\begin{array}{l}\text { Highest NNS intake } \\
\text { quantile v. none }\end{array}$ & $\mathrm{MD}+0.77 \mathrm{~kg} / \mathrm{m}^{2}$ (0.47 to 1.07$)$ & $\uparrow$ Gain & 50 & - \\
\hline Weight & $4(32405)$ & Continuous correlation & WMC $+0.06(0.05$ to 0.07$), 1^{2} 46 \%$ & $\uparrow$ Gain & 21,57 & 2 \\
\hline Gestational weight gain & $1(347)$ & $\begin{array}{l}\text { Highest v. lowest NNS } \\
\text { intake quantile }\end{array}$ & $\mathrm{MD}+2.5 \mathrm{~kg}(0.5$ to 4.5$)$ & $\uparrow$ Gain & 23 & - \\
\hline Weight gain $>1 \mathrm{~kg}$ & $1(7,194)$ & $\begin{array}{l}\text { Highest v. lowest NNS } \\
\text { intake quantile }\end{array}$ & OR 1.05 (0.93 to 1.19 ) & NS & 41 & - \\
\hline Waist circumference & $1(384)$ & $\begin{array}{l}\text { Daily v. no NNS } \\
\text { consumption }\end{array}$ & $\mathrm{MD}+2.27 \mathrm{~cm}$ (0.96 to 3.58$)$ & $\uparrow$ Gain & 18 & - \\
\hline Incident abdominal obesity & $1(5011)$ & $\begin{array}{l}\text { Highest v. lowest NNS } \\
\text { intake quantile }\end{array}$ & HR 1.59 (1.23 to 2.07 ) & $\uparrow$ Gain & 60 & \\
\hline Incident overweight/obesity & $3(7917)$ & $\begin{array}{l}\text { Highest v. lowest NNS } \\
\text { intake quantile }\end{array}$ & OR 1.84 ( 1.28 to 2.66 ), $1^{2} 0 \%$ & $\uparrow$ Risk & $22,50,59$ & $\mathrm{~S} 1 \ddagger$ \\
\hline Metabolic syndrome & $5(27914)$ & $\begin{array}{l}\text { Highest v. lowest NNS } \\
\text { intake quantile }\end{array}$ & RR 1.31 ( 1.23 to 1.40$), I^{2} 0 \%$ & $\uparrow$ Risk & $39,47,48,54,60$ & $\mathrm{~S} 2 \ddagger$ \\
\hline \multirow[t]{2}{*}{ Type 2 diabetes } & $4(221363)$ & Per daily serving of NNS & RR 1.03 (1.01 to 1.05 ), $120 \%$ & $\uparrow$ Risk & $24,42,56$ & $\mathrm{~S} 2 \ddagger$ \\
\hline & $9(400571)$ & $\begin{array}{l}\text { Highest v. lowest NNS } \\
\text { intake quantile }\end{array}$ & RR 1.14 (1.05 to 1.25$), I^{2} 52 \%$ & $\uparrow$ Risk & $\begin{array}{l}16,24,42,49 \\
55,56,58,60\end{array}$ & 2 \\
\hline Gestational diabetes & $1(13475)$ & $\begin{array}{l}\text { Highest v. lowest NNS } \\
\text { intake quantile }\end{array}$ & RR 0.87 (0.71 to 1.02 ) & NS & 44 & - \\
\hline Impaired glucose tolerance & $1(3728)$ & $\begin{array}{l}\text { No v. any NNS } \\
\text { consumption }\end{array}$ & HR 1.07 (0.91 to 1.26$)$ & NS & 48 & - \\
\hline Hypertension & $5(232630)$ & $\begin{array}{l}\text { Highest v. lowest NNS } \\
\text { intake quantile }\end{array}$ & HR 1.12 (1.08 to 1.13 ), $I^{2} 53 \%$ & $\uparrow$ Risk & $45,48,60$ & S4 $\ddagger$ \\
\hline Stroke & $2(128176)$ & $\begin{array}{l}\text { Highest v. lowest NNS } \\
\text { intake quantile }\end{array}$ & RR 1.14 (1.04 to 1.26 ), $120 \%$ & $\uparrow$ Risk & 40 & S4 \\
\hline Cardiovascular events $\dagger$ & $2(62178)$ & $\begin{array}{l}\text { Highest v. lowest NNS } \\
\text { intake quantile }\end{array}$ & RR 1.32 (1.15 to 1.52$), P^{2} 0 \%$ & $\uparrow$ Risk & 17,52 & S4 $\ddagger$ \\
\hline Coronary heart disease & $2(131403)$ & $\begin{array}{l}\text { Highest v. lowest NNS } \\
\text { intake quantile }\end{array}$ & RR 0.98 ( 0.90 to 1.07 ), $I^{2} 0 \%$ & NS & 46,51 & S4ł \\
\hline Chronic kidney disease & $1(14002)$ & $\begin{array}{l}\text { Highest v. lowest NNS } \\
\text { intake quantile }\end{array}$ & OR 0.80 (0.64 to 1.00$)$ & NS & 43 & - \\
\hline \multicolumn{7}{|c|}{ 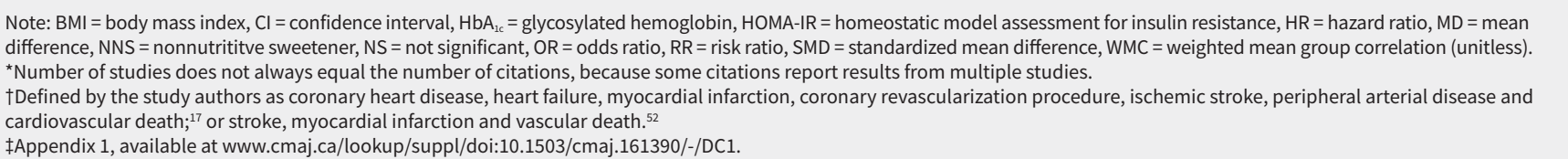 } \\
\hline
\end{tabular}




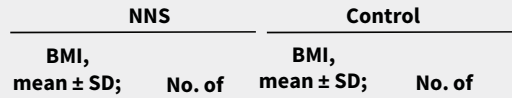

\section{Trial} $\mathrm{kg} / \mathrm{m}^{2}$ participants $\mathrm{kg} / \mathrm{m}^{2}$

$\begin{array}{llll}\text { Hsieh et al. } 2003^{36} & 23 \pm 2 & 82 & 23.6 \pm 2.4\end{array}$

Madjd et al. $2015^{20} \quad 30.6 \pm 3.8 \quad 32 \quad 30.6 \pm 2.8$

Ferri et al. $2006^{37} \quad 27.4 \pm 2.6$

Total

$6 \quad 25.8 \pm 2.8$

participants

$86-0.60(-1.27$ to 0.07$)$

30

$30 \quad 0.00(-1.65$ to 1.65$)$

120

122

$1.60(-1.46$ to 4.66$)$

$p^{2}=9 \%$

$22-0.37(-1.10$ to 0.36$)$

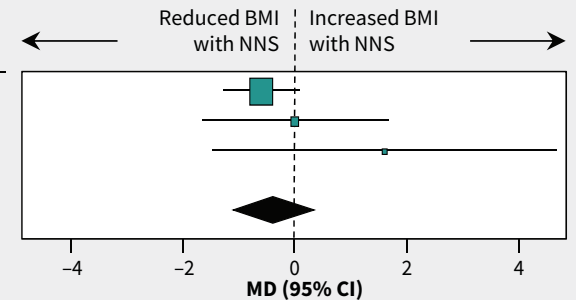

(B)

\begin{tabular}{|c|c|c|c|c|c|c|}
\hline Study (cohort) & $\begin{array}{c}\text { BMI change } \\
\text { correlation } \pm \mathrm{SE}\end{array}$ & Weight, \% & $\begin{array}{c}\text { Mean correlation } \\
(95 \% \mathrm{Cl})\end{array}$ & $\leftarrow$ & $\begin{array}{r}\text { Reduced BMI } \\
\text { with NNS }\end{array}$ & $\begin{array}{l}\text { Increased BMI } \\
\text { with NNS }\end{array}$ \\
\hline Gearon et al. 2014 (MCCS) ${ }^{15}$ & $0.043 \pm 0.008$ & 86.2 & $0.04(0.03$ to 0.06$)$ & & & \\
\hline Field et al. 2014 (GUTSII) ${ }^{14}$ & $0.063 \pm 0.02$ & 13.8 & $0.06(0.02$ to 0.10$)$ & & & \\
\hline Total & & 100.0 & $0.05(0.03$ to 0.06$)$ & & & \\
\hline$R^{2}=0 \%$ & & & & $\begin{array}{c}4 \\
-0.1\end{array}$ & $\begin{array}{c}1 \\
-0.05\end{array}$ & 0.05 \\
\hline
\end{tabular}

(C)

Weight change
mean \pm SD;

NS

Control

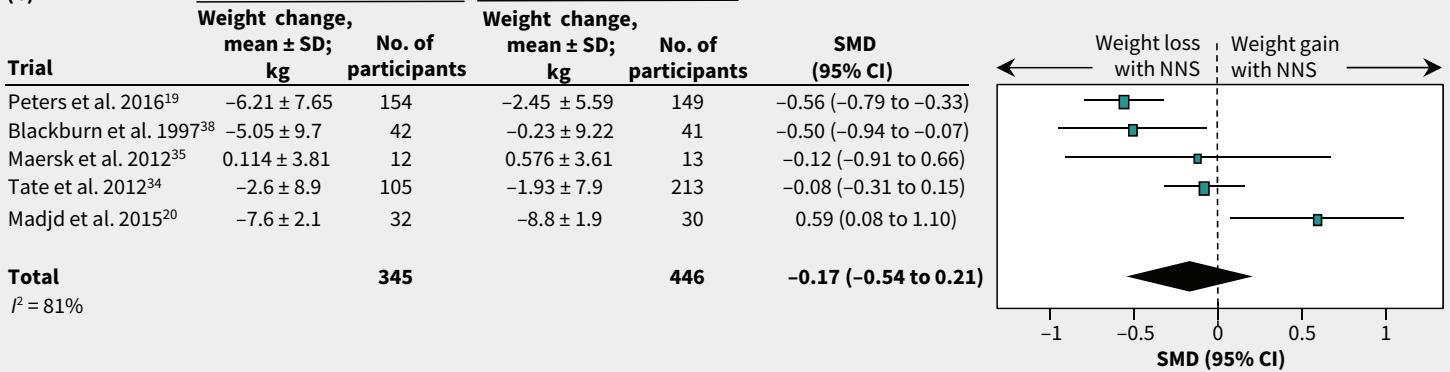

(D)

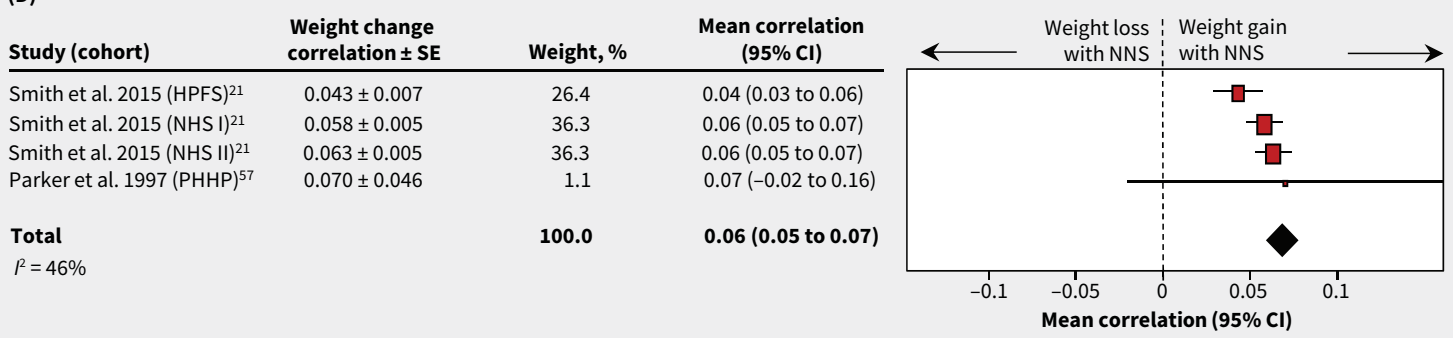

(E)

\section{Study (cohort)}

Pan et al. 2012 (NHS II) ${ }^{56}$

Bhupathiraju et al. 2013 (NHS I) ${ }^{42}$

Palmer et al. 2008 (BWHS) 55

Bhupathiraju et al. 2013 (HPFS) ${ }^{42}$

O'Connor et al. 2015 (EPIC-Norfolk) ${ }^{24}$

Nettleton et al. 2009 (MESA) 60

Stinson et al. 2013 (WHI) ${ }^{58}$

Fagherazzi et al. 2013 (EPIC-E3N) ${ }^{49}$

Sakurai et al. 2013 (Japan) ${ }^{16}$

Total $(95 \% \mathrm{CI})$

Heterogeneity: $\mathrm{Tau}^{2}=0.01 ; \mathrm{Chi}^{2}=16.60$ to $\mathrm{df}=8(P=0.03) ; I^{2}=52 \%$

Test for overall effect: $Z=3.08(P=0.002)$

\section{Type 2 diabetes}

$\log (\mathbf{R R})$

0.03

0.05

0.06

0.10

0.16

0.32

0.34

0.52

0.54

SE

SE

$0.06 \quad 18.6 \%$

$0.03 \quad 25.3 \%$

$0.13 \quad 8.1 \%$

$0.05 \quad 20.9 \%$

$0.12 \quad 9.0 \%$

$0.14 \quad 7.2 \%$

$0.26 \quad 2.6 \%$

$0.18 \quad 4.9 \%$

$0.22 \quad 3.4 \%$

$100.0 \%$

ght
$.6 \%$
$.3 \%$
$.1 \%$
$.9 \%$
$.0 \%$
$.2 \%$
$.6 \%$
$.9 \%$

$1.03(0.92$ to 1.16$)$

1.05 (0.99 to 1.11$)$

1.06 ( 0.82 to 1.37$)$

1.11 (1.00 to 1.22$)$

1.17 (0.93 to 1.48$)$

1.38 (1.05 to 1.81$)$

1.40 (0.84 to 2.34 )

1.68 ( 1.18 to 2.39$)$

1.72 (1.11 to 2.64 )

1.14 (1.05 to 1.25$)$
Lower type 2 diabetes $\quad$ Higher type 2 diabetes $\longleftarrow$ risk with NNS

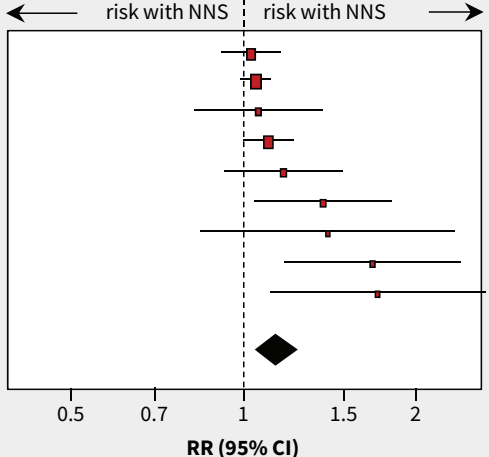

Figure 2: Forest plots of consumption of NNS and selected cardiometabolic health outcomes. (A) Differences in mean BMI between NNS consumption and control groups for RCTs. A value less than 0 represents reduced BMI with NNS consumption. (B) Correlaton of BMI change per unit of NNS intake for cohort studies. A value less than 0 represents a reduced BMI. (C) Standard mean differences in weight between NNS consumption and control groups for RCTs. A value less than 0.0 represents weight loss. (D) Correlation of weight change per unit NNS intake for cohort studies. A value less than 0 favours weight loss. (E) Incidence of type 2 diabetes for highest versus lowest quantiles of NNS intake in cohort studies. A value less than 1.0 represents a lower risk of type 2 diabetes. Additional outcomes are shown in Table 3, and Appendix 1, Figures S1-4. Squares represent effect estimates within each study, with $95 \% \mathrm{Cls}$ represented by horizontal lines. Square size is proportional to the weight of each study. Diamonds represent the weighted mean effect estimates. Cohort acronyms are defined in Table 2 . Note: $\mathrm{BMI}=$ body mass index, $\mathrm{Cl}=$ confidence interval, $\mathrm{MD}=$ mean difference, NNS $=$ nonnutritive sweetener, $\mathrm{RCT}$ = randomized controlled trial, $\mathrm{RR}=$ risk ratio, $\mathrm{SD}=$ standard deviation, $\mathrm{SE}=$ standard error, $\mathrm{SMD}=$ standardized mean difference. 
2 cohorts; 131403 participants) ${ }^{46,51}$ (Table 3 and Appendix 1, Figures S4B-4D).

\section{Publication bias}

Because of the limited number of studies, we could not assess publication bias for most outcomes, with the exception of type 2 diabetes. Although the pooled RR from 9 published studies that reported incident type 2 diabetes in high versus low consumers of nonnutritive sweeteners was significant (RR 1.14, 95\% Cl 1.05 to 1.23$)$, it was attenuated to $1.07(95 \% \mathrm{Cl} 0.97$ to 1.18$)$ after imputing missing studies (Appendix 1, Figure S5). This suggests potential publication bias that favours studies reporting a positive association between nonnutritive sweetener consumption and type 2 diabetes.

\section{Interpretation}

Evidence from small RCTs with short follow-up (median 6 mo) suggests that consumption of nonnutritive sweeteners is not consistently associated with decreases in body weight, BMI or waist circumference. However, in larger prospective cohort studies with longer follow-up periods (median $10 \mathrm{yr}$ ), intake of nonnutritive sweeteners is significantly associated with modest long-term increases in each of these measures. Cohort studies further suggest that consumption of nonnutritive sweeteners is associated with higher risks of obesity, hypertension, metabolic syndrome, type 2 diabetes, stroke and cardiovascular disease events; however, publication bias was indicated for type 2 diabetes, and there are no data available from RCTs to confirm these observations.

Previous reviews ${ }^{12,65}$ concluded that, although data from RCTs support weight-loss effects from sustained nonnutritive sweetener interventions, observational studies provide inconsistent results. Building on these findings, we included new studies ${ }^{14-24}$ and found that consumption of nonnutritive sweeteners was not generally associated with weight loss among participants in RCTs, except in long-term ( $\geq 12 \mathrm{mo}$ ) trials with industry sponsorship. In addition, we found that consumption of nonnutritive sweeteners was associated with modest long-term weight gain in observational studies. Our results also extend previous meta-analyses that showed higher risks of type 2 diabetes $^{32,33}$ and hypertension ${ }^{66}$ with regular consumption of nonnutritive sweeteners.

Our results highlight both the value and challenge of incorporating observational studies when examining the effect of realworld exposures on health outcomes that develop slowly over time. Although RCTs provide the highest quality of scientific evidence, they often fail to recapitulate chronic dietary exposures that are captured in decades-long cohort studies. However, it is not uncommon for hypotheses based on observational evidence to fail when tested in RCTs, ${ }^{67}$ and these data should therefore be interpreted with caution.

Strengths of our systematic review include use of a registered protocol and sensitive, peer-reviewed search strategy. We synthesized evidence from both RCTs and observational studies, assessed multiple cardiometabolic outcomes and focused on long-term effects.

\section{Limitations}

The main limitation of our review is the unavoidable grouping of exposure and outcome variables. We could not evaluate different types or formulations of nonnutritive sweeteners because most studies did not report this information, and we could not assess dose effects owing to the limited number of RCTs and the semiquantitative nature of the reporting of nonnutritive sweetener intake in cohort studies. In addition, some cardiometabolic outcomes could not be evaluated individually becuse of the way they were combined and reported in the original studies (e.g., "overweight and obesity," "cardiovascular events"). Finally, meta-analysis was not always possible because of reporting differences and the paucity of eligible studies.

The individual studies included in our review also have limitations. Most RCTs were at high risk of bias, and most cohort studies achieved only moderate quality scores. In the cohort studies, the ascertainment of exposure to nonnutritive sweeteners by self-report was likely incomplete, ${ }^{6}$ and the comparison of extreme intake quantiles may have yielded biased results. Furthermore, these studies evaluated consumption of artificially sweetened beverages before 2004; however, nonnutritive sweeteners are increasingly found in other foods, and consumption has increased considerably in recent years. ${ }^{6}$

Observational studies are also subject to confounding bias, particularly when the exposure (e.g., nonnutritive sweeteners) is a potential "treatment" for the outcomes under investigation. However, critical confounders (baseline body composition and diet quality) were largely accounted for in the included studies, and we limited confounding by reverse causation by including only prospective studies that documented intake of nonnutritive sweeteners before weight change and disease incidence.

Randomized controlled trials of nonnutritive sweetener interventions also have known limitations. ${ }^{68}$ All were relatively short in duration, and the majority were conducted as part of multifaceted weight loss programs in obese individuals, which does not address routine consumption of nonnutritive sweeteners by healthy individuals. In addition, some trials evaluated nonnutritive sweeteners in capsule form, which may alter their physiologic effects, while others were subject to potential bias from lack of blinding and industry sponsorship. Finally, several studies focused on BMI and waist circumference, which are imperfect indices of body composition, despite being established predictors of cardiovascular disease. ${ }^{69,70}$

\section{Conclusion}

Evidence from RCTs does not clearly support the intended benefits of nonnutritive sweeteners for weight management. In contrast, observational data suggest that routine consumption of nonnutritive sweeteners may be associated with a long-term increase in BMI and elevated risk of cardiometabolic disease; however, these associations have not been confirmed in experimental studies and may be influenced by publication bias. New studies are needed to compare different types and formulations of nonnutritive sweeteners, and to evaluate the net effect of substituting nonnutritive sweeteners for sugar. Improved assessment tools and biomarker approaches ${ }^{71}$ should be used to accu- 
rately capture consumption of nonnutritive sweeteners, and confounding bias must be carefully addressed. Given the widespread and increasing use of nonnutritive sweeteners, caution is warranted until the long-term risks and benefits of these products are fully characterized.

\section{References}

1. Ng M, Fleming T, Robinson M, et al. Global, regional, and national prevalence of overweight and obesity in children and adults during 1980-2013: a systematic analysis for the Global Burden of Disease Study 2013. Lancet 2014;384:766-81.

2. Johnson RK, Appel LJ, Brands M, et al. Dietary sugars intake and cardiovascular health: a scientific statement from the American Heart Association. Circulation 2009;120:1011-20.

3. Siervo M, Montagnese C, Mathers JC, et al. Sugar consumption and global prevalence of obesity and hypertension: an ecological analysis. Public Health Nutr 2014;17:587-96.

4. Gardner C, Wylie-Rosett J, Gidding SS, et al. Nonnutritive sweeteners: current use and health perspectives: a scientific statement from the American Heart Association and the American Diabetes Association. Diabetes Care 2012;35: 1798-808.

5. Sylvetsky AC, Welsh JA, Brown RJ, et al. Low-calorie sweetener consumption is increasing in the United States. Am J Clin Nutr 2012;96:640-6.

6. Sylvetsky AC, Rother KI. Trends in the consumption of low-calorie sweeteners. Physiol Behav 2016;164(Pt B):446-50.

7. Swithers SE. Artificial sweeteners produce the counterintuitive effect of inducing metabolic derangements. Trends Endocrinol Metab 2013;24:431-41.

8. Nettleton JE, Reimer RA, Shearer J. Reshaping the gut microbiota: Impact of low calorie sweeteners and the link to insulin resistance? Physiol Behav 2016; 164(Pt B):488-93.

9. Fowler SP. Low-calorie sweetener use and energy balance: results from experimental studies in animals, and large-scale prospective studies in humans. Physiol Behav 2016;164(Pt B):517-23.

10. Fitch C, Keim KS. Position of the academy of nutrition and dietetics: use of nutritive and nonnutritive sweeteners. J Acad Nutr Diet 2012;112:739-58.

11. Pereira MA. Diet beverages and the risk of obesity, diabetes, and cardiovascular disease: a review of the evidence. Nutr Rev 2013;71:433-40.

12. Miller PE, Perez V. Low-calorie sweeteners and body weight and composition: a meta-analysis of randomized controlled trials and prospective cohort studies. Am J Clin Nutr 2014;100:765-77.

13. Pan A, Hu FB. Question about a recent meta-analysis of low-calorie sweeteners and body weight. Am J Clin Nutr 2014;100:1604.

14. Field AE, Sonneville KR, Falbe J, et al. Association of sports drinks with weight gain among adolescents and young adults. Obesity (Silver Spring) 2014;22: 2238-43.

15. Gearon E, Peeters A, Hodge A, et al. The role of dietary and physical activity behaviours in educational differences in weight gain among Australian adults - the Melbourne Collaborative Cohort Study. Obesity Res Clin Practice 2014;8(S1):35-36.

16. Sakurai M, Nakamura K, Miura K, et al. Sugar-sweetened beverage and diet soda consumption and the 7-year risk for type 2 diabetes mellitus in middleaged Japanese men. Eur J Nutr 2014;53:251-8.

17. Vyas A, Rubenstein L, Robinson J, et al. Diet drink consumption and the risk of cardiovascular events: a report from the women's health initiative. J Gen Intern Med 2015;30:462-8.

18. Fowler SP, Williams K, Hazuda HP. Diet soda intake is associated with longterm increases in waist circumference in a biethnic cohort of older adults: the San Antonio Longitudinal Study of Aging. J Am Geriatr Soc 2015;63:708-15.

19. Peters JC, Beck J, Cardel M, et al. The effects of water and non-nutritive sweetened beverages on weight loss and weight maintenance: a randomized clinical trial. Obesity (Silver Spring) 2016;24:297-304.

20. Madjd A, Taylor MA, Delavari A, et al. Effects on weight loss in adults of replacing diet beverages with water during a hypoenergetic diet: a randomized, 24-wk clinical trial. Am J Clin Nutr 2015;102:1305-12.

21. Smith JD, Hou T, Hu FB, et al. A comparison of different methods for evaluating diet, physical activity, and long-term weight gain in 3 prospective cohort studies. J Nutr 2015;145:2527-34.

22. Lana A, Lopez-Garcia E, Rodriguez-Artalejo F. Consumption of soft drinks and health-related quality of life in the adult population. Eur J Clin Nutr 2015;69:1226-32.

23. Renault KM, Carlsen EM, Norgaard K, et al. Intake of sweets, snacks and soft drinks predicts weight gain in obese pregnant women: detailed analysis of the results of a randomised controlled trial. PLoS One 2015;10:e0133041.
24. O'Connor L, Imamura F, Lentjes MAH, et al. Prospective associations and population impact of sweet beverage intake and type 2 diabetes, and effects of substitutions with alternative beverages. Diabetologia 2015;58:1474-83.

25. Liberati A, Altman DG, Tetzlaff J, et al. The PRISMA statement for reporting systematic reviews and meta-analyses of studies that evaluate health care interventions: explanation and elaboration. J Clin Epidemiol 2009;62:e1-34.

26. Azad MB, Reid AE, Rabbani R, et al. Non-nutritive sweeteners and cardio-metabolic health: a systematic review and meta-analysis of randomized controlled trials and prospective cohort studies. London (UK): National Institute for Health Research - PROSPERO International prospective register of systematic reviews; 2015. Available: www.crd.york.ac.uk/PROSPERO/display_record. asp?ID=CRD42015019749 (accessed 2016 Jan).

27. Reid AE, Chauhan B, Rabbani R, et al. Early exposure to non-nutritive sweeteners and long-term metabolic health: a systematic review. Pediatrics 2015;137: $1-10$.

28. Becker BJ, Wu M-J. The synthesis of regression slopes in meta-analysis. Statist Sci 2007;22:414-29.

29. Higgins JPT, Green S, editors. Cochrane Handbook of Systematic Reviews of Interventions Version 5.1.0. [updated March 2011] edition. The Cochrane Collaboration; 2011.

30. Julian PTH, Douglas GA, Peter GGT, et al. The Cochrane Collaboration's tool for assessing risk of bias in randomized trials. BMJ 2011;343:d5928.

31. Wells GA, Shea B, O'Connell D, et al. The Newcastle-Ottawa Scale (NOS) for assessing the quality of nonrandomised studies in meta-analysis. Ottawa: The Ottawa Hospital Research Institute; 2013. Available: www.ohri.ca/programs/ clinical_epidemiology/oxford.asp

32. Imamura F, O'Connor L, Ye Z, et al. Consumption of sugar sweetened beverages, artificially sweetened beverages, and fruit juice and incidence of type 2 diabetes: systematic review, meta-analysis, and estimation of population attributable fraction. BMJ 2015;351:h3576.

33. Greenwood DC, Threapleton DE, Evans CE, et al. Association between sugarsweetened and artificially sweetened soft drinks and type 2 diabetes: systematic review and dose-response meta-analysis of prospective studies. Br J Nutr 2014;112:725-34.

34. Tate DF, Turner-McGrievy G, Lyons E, et al. Replacing caloric beverages with water or diet beverages for weight loss in adults: main results of the Choose Healthy Options Consciously Everyday (CHOICE) randomized clinical trial. Am J Clin Nutr 2012;95:555-63.

35. Maersk M, Belza A, Stodkilde-Jorgensen H, et al. Sucrose-sweetened beverages increase fat storage in the liver, muscle, and visceral fat depot: a 6-mo randomized intervention study. Am J Clin Nutr 2012;95:283-9.

36. Hsieh MH, Chan P, Sue YM, et al. Efficacy and tolerability of oral stevioside in patients with mild essential hypertension: a two-year, randomized, placebocontrolled study. Clin Ther 2003;25:2797-808.

37. Ferri LA, Alves-Do-Prado W, Yamada SS, et al. Investigation of the antihypertensive effect of oral crude stevioside in patients with mild essential hypertension. Phytother Res 2006;20:732-6.

38. Blackburn GL, Kanders BS, Lavin PT, et al. The effect of aspartame as part of a multidisciplinary weight-control program on short- and long-term control of body weight. Am J Clin Nutr 1997;65:409-18.

39. Barrio-Lopez MT, Martinez-Gonzalez MA, Fernandez-Montero A, et al. Prospective study of changes in sugar-sweetened beverage consumption and the incidence of the metabolic syndrome and its components: the SUN cohort. Br J Nutr 2013;110:1722-31.

40. Bernstein AM, de Koning L, Flint AJ, et al. Soda consumption and the risk of stroke in men and women. Am J Clin Nutr 2012;95:1190-9.

41. Bes-Rastrollo M, Sanchez-Villegas A, Gomez-Gracia E, et al. Predictors of weight gain in a Mediterranean cohort: the Seguimiento Universidad de Navarra Study 1. Am J Clin Nutr 2006;83:362-70.

42. Bhupathiraju SN, Pan A, Malik VS, et al. Caffeinated and caffeine-free beverages and risk of type 2 diabetes. Am J Clin Nutr 2013;97:155-66.

43. Bomback AS, Derebail VK, Shoham DA, et al. Sugar-sweetened soda consumption, hyperuricemia, and kidney disease. Kidney Int 2010;77:609-16.

44. Chen L, Hu FB, Yeung E, et al. Prospective study of pre-gravid sugar-sweetened beverage consumption and the risk of gestational diabetes mellitus. Diabetes Care 2009;32:2236-41.

45. Cohen L, Curhan G, Forman J. Association of sweetened beverage intake with incident hypertension. J Gen Intern Med 2012;27:1127-34.

46. de Koning L, Malik VS, Kellogg MD, et al. Sweetened beverage consumption, incident coronary heart disease, and biomarkers of risk in men. Circulation 2012;125:1735-41, S1.

47. Dhingra R, Sullivan L, Jacques PF, et al. Soft drink consumption and risk of developing cardiometabolic risk factors and the metabolic syndrome in middle-aged adults in the community. Circulation 2007;116:480-8. 
48. Duffey KJ, Steffen LM, Van Horn L, et al. Dietary patterns matter: diet beverages and cardiometabolic risks in the longitudinal Coronary Artery Risk Development in Young Adults (CARDIA) Study. Am J Clin Nutr 2012;95:909-15.

49. Fagherazzi G, Vilier A, Saes Sartorelli D, et al. Consumption of artificially and sugar-sweetened beverages and incident type 2 diabetes in the Etude Epidemiologique aupres des femmes de la Mutuelle Generale de l'Education Nationale-European Prospective Investigation into Cancer and Nutrition cohort. Am J Clin Nutr 2013;97:517-23.

50. Fowler SP, Williams K, Resendez RG, et al. Fueling the obesity epidemic? Artificially sweetened beverage use and long-term weight gain. Obesity (Silver Spring) 2008;16:1894-900.

51. Fung TT, Malik V, Rexrode KM, et al. Sweetened beverage consumption and risk of coronary heart disease in women. Am J Clin Nutr 2009;89:1037-42.

52. Gardener H, Rundek T, Markert M, et al. Diet soft drink consumption is associated with an increased risk of vascular events in the Northern Manhattan Study. J Gen Intern Med 2012;27:1120-6.

53. InterAct Consortium, Romaguera D, Norat T, Wark PA, et al. Consumption of sweet beverages and type 2 diabetes incidence in European adults: results from EPIC-InterAct. Diabetologia 2013;56:1520-30.

54. Lutsey PL, Steffen LM, Stevens J. Dietary intake and the development of the metabolic syndrome: the Atherosclerosis Risk in Communities study. Circulation 2008;117:754-61.

55. Palmer JR, Boggs DA, Krishnan S, et al. Sugar-sweetened beverages and incidence of type 2 diabetes mellitus in African American women. Arch Intern Med 2008; 168:1487-92.

56. Pan A, Malik VS, Schulze MB, et al. Plain-water intake and risk of type 2 diabetes in young and middle-aged women. Am J Clin Nutr 2012;95:1454-60.

57. Parker DR, Gonzalez S, Derby CA, et al. Dietary factors in relation to weight change among men and women from two southeastern New England communities. Int J Obes Relat Metab Disord 1997;21:103-9.

58. Stinson LJ, Bansari A, Quddus A, et al. Association between artificially sweetened beverages and the incidence of type 2 diabetes in postmenopausal women [abstract MP95]. Circulation 26 Mar 2013;127.

59. Haines J, Neumark-Sztainer D, Wall M, et al. Personal, behavioral, and environmental risk and protective factors for adolescent overweight. Obesity (Silver Spring) 2007;15:2748-60.
60. Nettleton JA, Lutsey PL, Wang Y, et al. Diet soda intake and risk of incident metabolic syndrome and type 2 diabetes in the Multi-Ethnic Study of Atherosclerosis (MESA). Diabetes Care 2009;32:688-94.

61. de Koning L, Malik VS, Rimm EB, et al. Sugar-sweetened and artificially sweetened beverage consumption and risk of type 2 diabetes in men. Am J Clin Nutr 2011; 93:1321-7.

62. Winkelmayer WC, Stampfer MJ, Willett WC, et al. Habitual caffeine intake and the risk of hypertension in women. JAMA 2005;294:2330-5.

63. Colditz GA, Willett WC, Stampfer MJ, et al. Patterns of weight change and their relation to diet in a cohort of healthy women. Am J Clin Nutr 1990;51: 1100-5.

64. Schulze MB, Manson JE, Ludwig DS, et al. Sugar-sweetened beverages, weight gain, and incidence of type 2 diabetes in young and middle-aged women. JAMA 2004;292:927-34.

65. Rogers PJ, Hogenkamp PS, de Graaf C, et al. Does low-energy sweetener consumption affect energy intake and body weight? A systematic review, including meta-analyses, of the evidence from human and animal studies. Int $\mathrm{J}$ Obes (Lond) 2016;40:381-94.

66. Cheungpasitporn W, Thongprayoon C, Edmonds PJ, et al. Sugar and artificially sweetened soda consumption linked to hypertension: a systematic review and meta-analysis. Clin Exp Hypertens 2015;37:587-93.

67. Maki KC, Slavin JL, Rains TM, et al. Limitations of observational evidence: implications for evidence-based dietary recommendations. Adv Nutr 2014;5:7-15.

68. Sylvetsky AC, Blau JE, Rother KI. Understanding the metabolic and health effects of low-calorie sweeteners: methodological considerations and implications for future research. Rev Endocr Metab Disord 2016;17:187-94.

69. Ortega FB, Sui X, Lavie CJ, et al. Body mass index, the most widely used but also widely criticized index: Would a criterion standard measure of total body fat be a better predictor of cardiovascular disease mortality? Mayo Clin Proc 2016; 91:443-55.

70. Lee WS. Body fatness charts based on BMI and waist circumference. Obesity (Silver Spring) 2016;24:245-9.

71. Logue C, Dowey LC, Strain JJ, et al. The potential application of a biomarker approach for the investigation of low-calorie sweetener exposure. Proc Nutr Soc 2016;75:216-25.
Competing interests: Jonthan McGavock has received speaker fees from Medtronic. No other competing interests were declared.

This article has been peer reviewed.

Affiliations: George \& Fay Yee Centre for Healthcare Innovation (Azad, Abou-Setta, Chauhan, Rabbani, Lys, Copstein, Mann, Jeyaraman, Fiander, Zarychanski); Children's Hospital Research Institute of Manitoba (Azad, Chauhan, McGavock, Wicklow); Department of Pediatrics and Child Health (Azad, McGavock, Wicklow); Department of Community Health Sciences (Abou-Setta); College of Pharmacy (Chauhan); Max Rady College of Medicine (Reid); Department of Human Nutritional Sciences (Azad, MacKay); Department of Internal Medicine (Zarychanski), University of Manitoba; Department of Hematology and Medical Oncology, CancerCare Manitoba (Zarychanski), Winnipeg, Man.

Contributors: Meghan Azad conceptualized and coordinated the study. Meghan Azad and Ashleigh Reid drafted the initial protocol.
Michelle Fiander developed the search strategy. Ashleigh Reid, Justin Lys, Leslie Copstein, Amrinder Mann and Maya Jeyaraman screened citations and assessed studies for eligibility. Rasheda Rabbani, Bhupendrasinh Chauhan, Ahmed Abou-Setta, Leslie Copstein and Meghan Azad extracted data. Meghan Azad, Justin Lys, Leslie Copstein and Bhupendrasinh Chauhan performed quality assessments. Rasheda Rabbani performed statistical analyses. Dylan MacKay, Jon McGavock and Brandy Wicklow provided content expertise in nutrition and metabolic health. Ryan Zarychanski, Bhupendrasinh Chauhan and Ahmed Abou-Setta provided methodologic expertise in knowledge synthesis and resolved disagreements regarding study eligibility or quality assessments. Dylan MacKay, Jon McGavock, Brandy Wicklow, Ryan Zarychanski, Bhupendrasinh Chauhan and Ahmed Abou-Setta critically reviewed the manuscript for important intellectual content. All of the authors gave final approval of the version to be published and agreed to be accountable for all aspects of the work.
Funding: No funding was specifically obtained for this study. Ryan Zarychanski received a New Investigator Award from the Canadian Institutes of Health Research (CIHR). Jon McGavock holds the CIHR Applied Public Health Chair in Resilience and Childhood Obesity. CIHR had no role in the design, conduct or reporting of the study.

Acknowledgements: The authors thank information specialist consultant Becky Skidmore for her critical review of the search strategy. They also thank the following study authors for contributing additional unpublished data: Maira Bes-Rastrollo and colleagues (Seguimiento Universidad de Navarra [SUN] cohort), Emma Gearon and colleagues (Melbourne Collaborative Cohort Study [MCCS] cohort), and Esther Lopez-Garcia and colleagues (Study on Nutrition and Cardiovascular Risk in Spain [ENRICA] cohort).

\section{Accepted: Mar. 10, 2017.}

Correspondence to: Meghan Azad, meghan.azad@umanitoba.ca 